\title{
Anticipation- and Error-related EEG Signals during Realistic Human-Machine interaction: A Study on Visual and Tactile Feedback
}

\author{
Ricardo Chavarriaga, Xavier Perrin, Roland Siegwart and José del R. Millán
}

\begin{abstract}
The exploitation of EEG signatures of cognitive processes can provide valuable information to improve interaction with brain actuated devices. In this work we study these correlates in a realistic situation simulated in a virtual reality environment. We focus on cortical potentials linked to the anticipation of future events (i.e. the contingent negative variation, CNV) and error-related potentials elicited by both visual and tactile feedback. Experiments with 6 subjects show brain activity consistent with previous studies using simpler stimuli, both at the level of ERPs and single trial classification. Moreover, we observe comparable signals irrespective of whether the subject was required to perform motor actions. Altogether, these results support the possibility of using these signals for practical brain machine interaction.
\end{abstract}

\section{INTRODUCTION}

A large body of research has focused on the study of brain correlates of different perceptual and cognitive processes. In particular, EEG signatures of anticipation [1] and error awareness [2] may be exploited by brain-machine interfacing (BMI) systems to complement traditional control paradigms (e.g. based on kinesthetic movement imagination) in order to enhance human-machine interaction. As an example, it has been shown that error-related EEG potentials (ErrP) can be used to correct BMI misclassification [3] or as a teaching signal to improve performance [4], [5].

However, most of these studies are performed in wellcontrolled laboratory conditions using simple visual or auditory stimulus. Therefore, characterization of these correlates in real life situations -a key issue for practical applicationsremains to be elucidated. More recently, the use of virtual reality has been proposed as a mean to overcome this limitation in both neuroscience [6] and BMI [7] research. Following this line we use a virtual reality setup to study these correlates during a realistic semi-autonomous navigation task. We focus in the following signals:

1) Anticipation related potentials: Previous studies have shown the development of a slow negative wave in frontalcentral areas during the time interval between two events, corresponding to a warning and imperative stimulus respectively [1]. This signal, termed contingent negative variation (CNV) is supposed to reflect the anticipation of the upcoming event. Some previous studies have suggested its presence in more realistic experimental paradigms [8], as well as its potential use in BMI applications [9], [10].

R. Chavarriaga, and J. del R. Millán are with EPFL, Chair on NonInvasive Brain-Computer Interface (CNBI), CH-1015 Lausanne, Switzer-

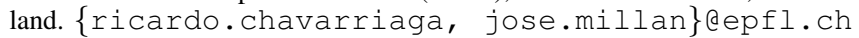

$X$. Perrin and R. Siegwart are with ETH Zurich, ASL Lab, CH-8092 Zurich, Switzerland. xavier.perrinda3.epfl.ch, rsiegwarteethz.ch
2) Error related potentials: We also study EEG activity elicited by correct and erroneous feedback information. These signals have been reported to appear in frontocentral medial electrodes between $200 \mathrm{~ms}$ and $600 \mathrm{~ms}$ after stimulus onset. Given the importance of error processing for learning, ErrPs are of great interest in both neurophysiological studies [11] and BMI applications [4], [3], [12].

Moreover, effective BMI systems are required to work in closed loop and efficiently convey information to the user. Although some information can already be perceived through the overt behavior of the controlled device (e.g. movement of a robotic arm or wheelchair), additional information (e.g. the decoded command before its execution) can also be useful to facilitate control or avoid wrong decisions. We study the use of visual and tactile feedback to convey such information and report the EEG signals elicited by each of them. This extends previous work evaluating the user preferences on this regard [13].

In this work we study anticipation and error-related potentials elicited by both visual and tactile feedback during a navigation tasks in a realistic environment. Furthermore, we compare ERPs and single-trial classification in conditions with and without user motor responses. The experimental protocol is presented in Section II. This section also describes the EEG recording and processing techniques. Results on both anticipatory and error related potentials are presented in Section III, followed by the conclusions (Section IV).

\section{Methods}

\section{A. Experimental protocol}

We adopt an experimental protocol used in a previous study on multimodal feedback for brain-based semiautonomous navigation [13]. Six male subjects (mean age $27.67 \pm 1.75$ ) took part in the experiment, all of them have normal or corrected to normal vision and gave informed written consent to participate in the experiment.

In order to have realistic stimuli, we run the experiment in a large-scale projection room. The system is composed of two projectors Christie Mirage 5000 (1280x1024, activestereo capability). The projection surface has a height of $3.87 \mathrm{~m}$ and a radius of $4.55 \mathrm{~m}$, with a total angle of $150^{\circ}$. The bottom part has a curve with a radius of $1.5 \mathrm{~m}$ approximately.

Subjects are seated in front of the projection system, the visual scene corresponds to the interior of a maze as if they were on a mobile robot moving across the maze (Figure 1(a)). The robot moves autonomously and subjects are asked to monitor the decisions it takes at each intersection. Whenever the robot arrives at an intersection, it stops and proposes 


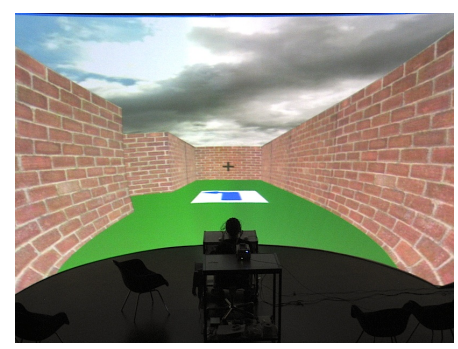

(a)

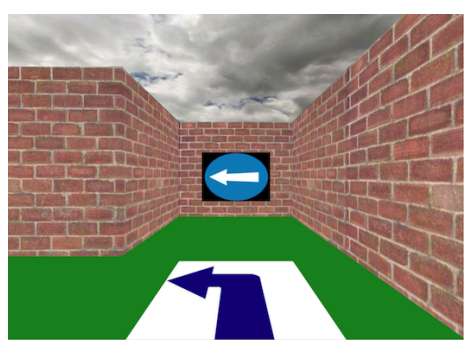

(b)

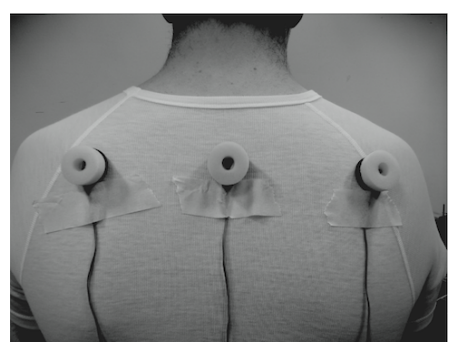

(c)

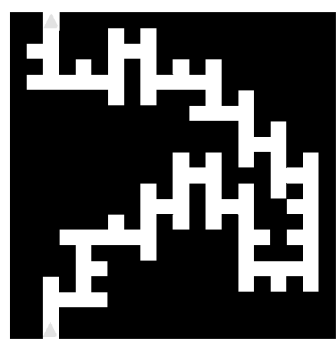

(d)

Fig. 1. (a) Experimental environment consisting of a cylindrical wide projection screen covering the subject's visual field. The subject and EEG system can be seen in the center. The curvature of the projection surface can be observed in the bottom of the image. (b) Example of (correct) visual feedback. (c) Placement of the stimulators used to provide tactile feedback. (d) Example of one of the mazes used during the experiment.

an action (i.e. turn left, turn right or move forward) using visual or tactile feedback. The subject should assess whether the proposition is correct or not. Inside the maze, the correct trajectory is indicated by arrows drawn on the floor. After the feedback presentation the robot waits for one second before resuming its path. It should be noticed that the robot always follows the correct trajectory towards the exit of the maze.

Before starting the experiment, subjects performed a calibration routine to ensure that they correctly perceive both types of feedback information. Each feedback type was delivered as follows:

1) Visual feedback: An icon containing an arrow (pointing left, right, or $u p$ ), similar to a traffic sign, is shown in the center of the screen (c.f. Figure 1(b)).

2) Tactile feedback: Vibrotactile actuators (Engineering Acoustics, INC., Fl, USA, http://www.eaiinfo.com) located at the left, center and right side of the upper back of the user are activated according to the robot decision (Figure 1(c)). The electromagnetic devices are activated using a bluetooth interface to provide a vibrating stimulation $(200 \mathrm{~Hz})$ during 50 milliseconds.

For each feedback type, two conditions were recorded. In the first condition (Keypress) the subject was asked to press a key whenever the system proposition was erroneous. Figure 2 shows the reaction time (i.e. time between the stimulus and the key press) is around $550 \mathrm{~ms}$. Consistent with a previous study subjects tend to respond slightly faster to visual feedback [13].

In the second condition (Monitoring), the subject evaluates the system's performance without executing any motor action (as it would be the case for BMI applications). Comparison across the two conditions allows to evaluate the influence of motor preparation and action on the observed EEG signals.

For each condition and feedback type, the subject navigates through three different mazes, each one containing 33 binary intersections (i.e. 99 trials in total per case). An example of one of these mazes is shown in Figure 1(d). The robot controller was set up so as to propose erroneous directions in $40 \%$ of the trials. The speed of the robot was set so as to spend three seconds between two successive intersections.

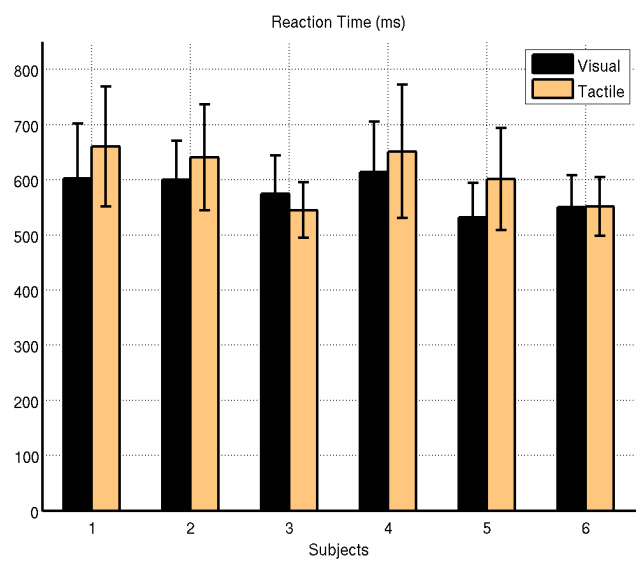

Fig. 2. Reaction time, Keypress condition. It corresponds to the time (in $\mathrm{ms}$ ) required to press the key after erroneous (visual or tactile) feedback.

\section{B. EEG recordings}

During the experiment we recorded EEG activity in order to analyze two types of event-related potentials: the CNV signal related to the anticipation of upcoming events (in this case, the new crossing at the end of each path segment), and the error-related potentials elicited by the feedback (i.e. proposed movement directions) provided by the system.

Data was recorded with a Biosemi acquisition system (http://www.biosemi.nl) with a sampling rate of $512 \mathrm{~Hz}$. We used 64 electrodes according to the extended 10/20 international system, re-referenced to the common average reference. External triggers were sent to the acquisition board of the EEG system by the experimental software to timestamp relevant events (i.e., experiment start/stop, feedback delivery, and user's response).

1) Contingent Negative Variation: In the current paradigm, as the user navigates through a corridor he can anticipate the feedback presentation at the next crossing. Although there is no explicit warning stimuli, several cues tell the user about the upcoming crossing. Therefore, we can hypothesize the development of CNV signals between two consecutive crossings.

EEG data was low-pass filtered with a cutoff frequency of $5 \mathrm{~Hz}$, and smoothed using a moving average window of 200 

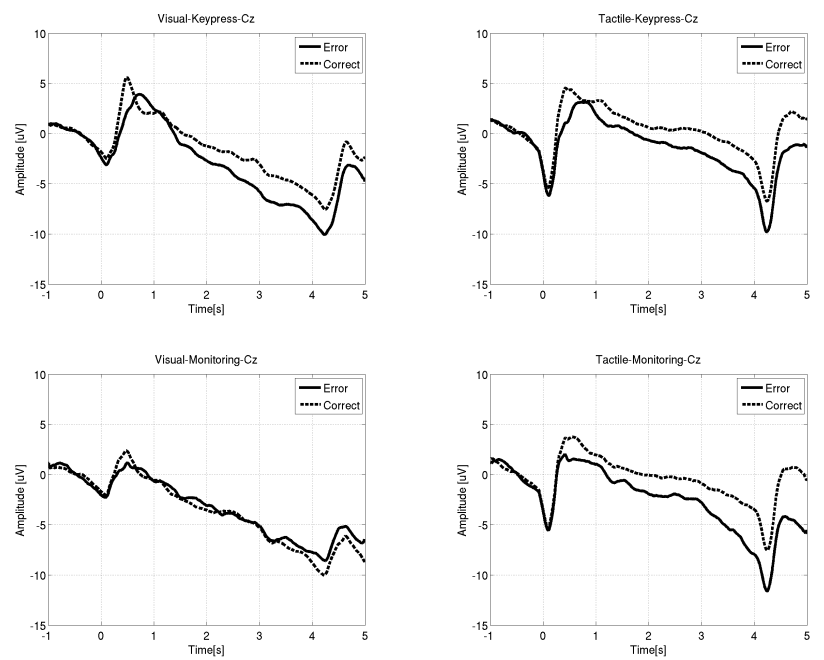

Fig. 3. Contingent negative variation. Grand Average ERP, Cz Electrode. A negative deflection develops between two consecutive intersection. Feedback is presented at time $t=0$ and $t=4$ respectively. The continuous line corresponds to epochs where erroneous feedback was provided at $t=0$, while the dotted line corresponds to correct feedback at that point. Left, Visual feedback- Right, Tactile feedback. Top, Condition 1, Keypress. Bottom, Condition 2, Monitoring

ms. We report the signal at the vertex ( $\mathrm{Cz}$ electrode) between two consecutive crossings, corresponding to a time window of 4 seconds ( 1 second of wait after feedback plus 3 seconds of movement along the corridor). Each epoch was baseline corrected to the mean value in a 1s window before feedback onset. Since several studies have reported behavioral changes after erroneous actions or feedback [14], [15] we also assess possible modulations of the CNV signals depending on the correctness of the presented feedback.

2) Error related potentials: To analyze error related potentials, a $2-10 \mathrm{~Hz}$ band-pass filter was applied on the EEG data before being segmented into epochs corresponding to each maze intersection. Each epoch lasts until $1000 \mathrm{~ms}$ after the feedback onset and is baseline corrected with the average activity in the $100 \mathrm{~ms}$ window prior to the feedback delivery.

Following previous work, we use the activity of the $\mathrm{Fz}$ and $\mathrm{FCz}$ electrodes to classify the activity elicited by correct and erroneous feedback [4], [3]. The signal in the time window from $100 \mathrm{~ms}$ to $500 \mathrm{~ms}$ after the stimulus onset was downsampled to $32 \mathrm{~Hz}$ and feed into the classifier. We train a Linear discriminant (LDA) classifier separately per subject, condition and type of feedback. The classification performance was assessed using 10-fold cross validation.

\section{RESULTS}

\section{A. Contingent Negative Variation}

Figure 3 shows the grand average ERP on the $\mathrm{Cz}$ electrode between two consecutive crossings. Time $t=0$ corresponds to the feedback onset at the beginning of a maze segment. After 1 second, the robot starts to move and takes three seconds to reach the next intersection where new feedback is provided. A feedback-evoked positive inflection can be clearly seen about $200 \mathrm{~ms}$ after the stimulus onset for both modalities $(\mathrm{t}=0$
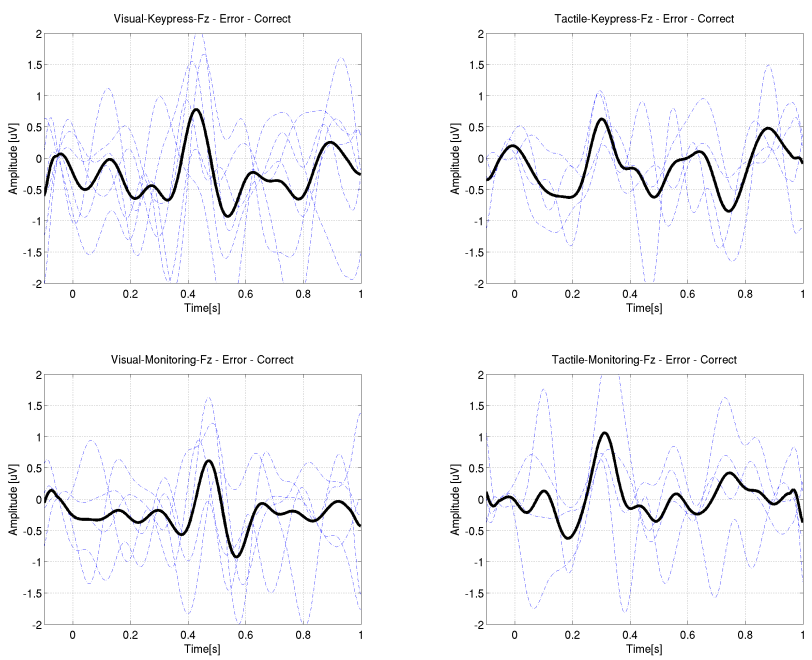

Fig. 4. Error-related potentials. Grand Average ERP, Fz electrode: Error minus correct condition. Time $\mathrm{t}=0$ corresponds to the feedback onset. Left, Visual feedback. Right, Tactile feedback. Top, Condition 1, Keypress. Bottom, Condition 2, Monitoring. Thin lines correspond to the ErrP of individual subjects. The Thick line shows the average across subjects

and $\mathrm{t}=4 \mathrm{~s}$ ). Moreover, a sharp, larger negative peak appears after delivery of tactile feedback.

A slow negative deflection, consistent with the reported CNV appears during the time between the two crossings in the maze. It appears in both conditions for all types of feedback. Interestingly, this negativity is larger after erroneous feedback is delivered (continuous traces), except when visual feedback is used in the Monitoring condition. Statistical differences $(p<0.05)$ where found in the window [3500 4000] for three of the subjects. Various studies proposed behavioral adjustments made after errors as evidence of subjects increasing their attention to the task [2], [16]. Accordingly, these differences in the CNV may be due to attentional changes after wrong propositions.

\section{B. Error-related EEG potentials}

Error-related signals similar to previous studies can be observed in frontocentral areas for all types of feedback in both conditions. We report the grand average difference ERP (i.e. erroneous minus correct trials) for electrode $\mathrm{Fz}$ across all subjects in Figure 4. The positive peak of the ErrP appears shortly after $400 \mathrm{~ms}$ after visual feedback and around $300 \mathrm{~ms}$ for tactile feedback, irrespective of whether a motor action is required. Interestingly, the timing difference in the peak ErrP is opposite to differences in the reaction time during the Keypress condition (c.f. Figure 2).

Figure 5 shows the ErrP classification performance in the ROC (Receiver Operating characteristic) space. Each continuous line in a plot depicts the mean accuracy (10-fold cross-validation) for one subject. The $y$-axis represents the true positive rate (TPR; amount of correct trials classified as such); while the $\mathrm{x}$-axis corresponds to the false negative rate (FNR; amount of misclassified error trials). A perfect classifier will appear on the upper left corner of the plot 

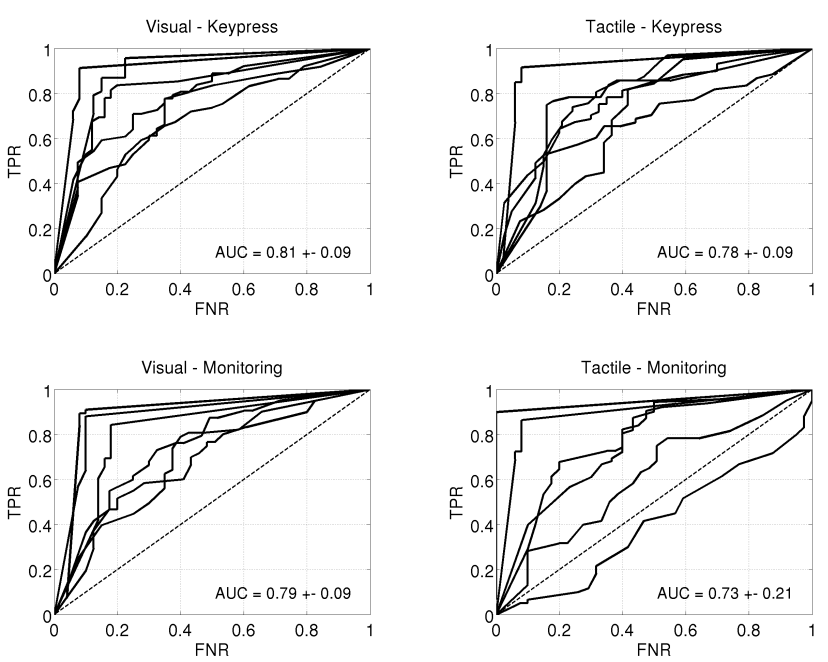

Fig. 5. Classification performance in the ROC space. X-axis. False negative rate. Y-axis: True positive rate. Left, Visual feedback- Right, Tactile feedback.Top, Condition 1, Keypress. Bottom, Condition 2, Monitoring

$(\mathrm{TPR}=1 ; \mathrm{FNR}=0)$. The dotted diagonal line corresponds to random performance. The figure also reports the area under the ROC (AUC) curve averaged across subjects. It can be seen that erroneous and correct trials can be classified above random level for all cases, with the exception of one subject in the Monitoring condition with tactile feedback. In general, higher performance is achieved for the Keypress condition, where a motor action is required after erroneous feedback.

\section{CONCLUSION}

We report EEG correlates of anticipation and error processing during navigation in virtual environments. The experiment was performed in a large-scale projection system as a step towards the use of these signals in real applications. Fuurthermore, we tested two types of feedback to convey information to the user and compare the results with and without motor responses.

The measured signals are consistent with previous studies. This confirmis that they can be observed in realistic interactive situations, despite the complexity of the stimuli perceived by the user. Moreover, similar correlates were found for all feedback modalities and response conditions. Importantly, their appearance when no motor response is required, as well as the results of single trial classification of ErrPs, supports their potential use for brain-machine interaction.

Further work is being performed to test online classification of these signals in simulated and real devices [17], [18]. In addition, source localization and connectivity methods will be explored to assess the information flow from sensory specific areas towards unified error-monitoring structures (e.g. Anterior cingulate cortex) or motor areas [19], [20].

\section{ACKNOWLEDGEMENTS}

This work was supported by the EU project BACS FP6IST-027140 and the Swiss National Science Foundation (SNSF) National Center of Competence in Research (NCCR)
Robotics. Virtual mazes were created using the Virtual Environments Library, veLib (http://velib.kyb.mpg.de).

\section{REFERENCES}

[1] W. G. Walter, R. Cooper, V. J. Aldridge, W. C. McCallum, and A. L. Winter, "Contingent negative variation: An electric sign of sensorimotor association and expectancy in the human brain." Nature, vol. 203, pp. 380-4, Jul 251964.

[2] S. F. Taylor, E. R. Stern, and W. J. Gehring, "Neural systems for error monitoring: Recent findings and theoretical perspectives." Neuroscientist, vol. 13, no. 2, pp. 160-172, Apr 2007.

[3] P. W. Ferrez and J. Millán, "Error-related EEG potentials generated during simulated brain-computer interaction," IEEE Transactions on Biomedical Engineering, vol. 55, pp. 923-929, 2008.

[4] R. Chavarriaga and J. Millán, "Learning from EEG error-related potentials in noninvasive brain-computer interfaces." IEEE Trans Neural Syst Rehabil Eng, vol. 18, no. 4, pp. 381-388, Aug 2010.

[5] I. Iturrate, L. Montesano, and J. Minguez, "Robot reinforcement learning using EEG-based reward signals," in IEEE International Conference on Robotics and Automation (ICRA), Alaska (USA), 2010.

[6] C. J. Bohil, B. Alicea, and F. A. Biocca, "Virtual reality in neuroscience research and therapy." Nat Rev Neurosci, vol. 12, no. 12, pp. $752-762,2011$.

[7] R. Leeb, D. Friedman, G. R. Müller-Putz, R. Scherer, M. Slater, and G. Pfurtscheller, "Self-paced (asynchronous) BCI control of a wheelchair in virtual environments: a case study with a tetraplegics," Computational Intelligence and Neuroscience, vol. 2007, p. 79642, 2007.

[8] J. D. Bayliss, "A flexible brain-computer interface," $\mathrm{PhD}$ thesis, University of Rochester, Rochester, New York, 2001.

[9] G. Gangadhar, R. Chavarriaga, and J. d. R. Millán, "Fast recognition of anticipation related potentials," IEEE Tran Biomed Eng, vol. 56, no. 4, pp. 1257-60, 2009.

[10] G. Garipelli, R. Chavarriaga, and J. d. R. Millán, "Single trial recognition of anticipatory slow cortical potentials: The role of spatiospectral filtering," in Proceedings of the 5th International Conference on Neural Engineering, 2011.

[11] S. Hoffmann and M. Falkenstein, "Predictive information processing in the brain: Errors and response monitoring." Int J Psychophysiol, Dec 2011.

[12] M. Spüler, M. Bensch, S. Kleih, W. Rosenstiel, M. Bogdan, and A. Kübler, "Online use of error-related potentials in healthy users and people with severe motor impairment increases performance of a P300-BCI." Clin Neurophysiol, Jan 2012.

[13] X. Perrin, R. Chavarriaga, C. Ray, R. Siegwart, and J. Millán, "A comparative psychophysical and EEG study of different feedback modalities for human-robot interaction," in ACM/IEEE Conf on Human-Robot Interaction HRIO8, Amsterdam, Netherlands, 2008.

[14] P. M. A. Rabbitt, "Errors and error correction in choice reaction tasks," J Exp Psychol Gen, vol. 71, pp. 264-72, 1966.

[15] M. X. Cohen and C. Ranganath, "Reinforcement learning signals predict future decisions." J Neurosci, vol. 27, no. 2, pp. 371-378, Jan 2007.

[16] S. Debener, M. Ullsperger, M. Siegel, K. Fiehler, D. Y. von Cramon, and A. K. Engel, "Trial-by-trial coupling of concurrent electroencephalogram and functional magnetic resonance imaging identifies the dynamics of performance monitoring." J Neurosci, vol. 25, no. 50, pp. 11 730-11 737, Dec 2005.

[17] X. Perrin, R. Chavarriaga, F. Colas, R. Siegwart, and J. Millán, "Braincoupled interaction for semi-autonomous navigation of an assistive robot." Robotics and Autonomous Systems, vol. 58, no. 12, pp. 1246$1255,2010$.

[18] Z. Khaliliardali, R. Chavarriaga, L. Gheorghe, and J. d. R. Millán, "Detection of anticipatory brain potentials during car driving," in 34th International Conference of the IEEE Engineering in Medicine and Biology Society (EMBC'12), 2012.

[19] M. K. Goel, R. Chavarriaga, and J. d. R. Millán, "Cortical Current Density vs. surface EEG for Event-Related Potential-based BrainComputer Interface," in 5th International IEEE EMBS Conference on Neural Engineering, 2011.

[20] H. Zhang, R. Chavarriaga, M. K. Goel, L. Gheorghe, and J. d. R. Millán, "Improve the performance of error monitoring by brain connectivity features," in 34th International Conference of the IEEE Engineering in Medicine and Biology Society (EMBC'12), 2012. 